\section{Intensification of the Latent (or Developable) Image.}

I T was shown recently by Wightman, Trivelli, and Sheppard (of the Research Laboratory of the Eastman Kodak Company) that when single-layer plates were given a very short exposure to light and then treated with a very dilute solution of hydrogen peroxide, that the resulting developability was greater than the sum of the two separate effects. It was suggested that a part of the light-exposure effect was undevelopable, and that the peroxide, besides producing its own developable image, carried over the undevelopable part of the other into the developable condition. This is the phenomenon that is called the " intensification of the latent image."

Messrs. Wightman and Quirk (Jour. Franklin Institute, Feb. 1927) have extended this work, using ordinary plates and films. Their method is to give a graduated exposure and to divide the plate into three strips-(1) developed straight away, (2) developed after soaking in a very dilute $(0.004$ to 0.016 per cent.) solution of hydrogen peroxide, and (3) exactly like (2), but using plain water. The concentration of the peroxide and the time of treatment were varied, and silver nitrate was used instead of the peroxide. They find that hydrogen peroxide and silver nitrate, and perhaps some other noble metal salts, acting on a photographic plate after exposure and before development, increase the developability of the lightexposed portion above its normal developability and in a greater proportion than the unexposed part. The effect is approximately equivalent in degree to that produced by a brief flash exposure of the plate to light before or after the principal exposure. Other circumstances being equal, the effect is smaller on plates of medium speed, and not detectable at all on 'process' (slow) plates or plates that have been desensitised by chromic acid, unless the concentration of the hydrogen peroxide or the time of treatment is considerably increased. By this method it is possible to detect one part of hydrogen peroxide in ten million parts of water to within 20 or 30 per cent. It is considered that the process is not suitable for general photographic work because it needs too much care in its application.

\section{University and Educational Intelligence.}

London.-The following courses of free public lectures are announced :-

At University College, at 4.30, on May 3, 4, and 6, "Sensation and the Sensory Pathway," by Prof. J. S. B. Stopford ; at King's College, at 5, on May 3, 10, 17, and 24, "Autonomic Nervous System," by Prof. R. J. S. McDowall. At the Imperial College of Science--Royal School of Mines, at 5.30, on May 10, 17, and 24, "Problems of the Respiration of Plants," by Dr. F. F. Blackman. No tickets will be required.

The Rev. L. Van Vestraut, who has for several years been chief assistant in the Testing Department at Faraday House Electrical Engineering College, has been appointed Registrar. Mr. Vestraut is an old student of the College, and was head student of his year. Mr. F. A. Bell has been appointed Secretary of the College.

THE New Education Fellowship will hold its fourth international conference at Locarno on Aug. 3-15 next. The general theme is to be the true meaning of freedom in education; Prof. Pierre Bovet of Geneva will be president. The freedom desired is that which comes from inner control, not external restraint, and the conference will seek to discover the basic principles underlying the many novel systems such as the methods called Winnetka, Project, Mackinder, Decroly, Montessori, Dalton, and Howard. Secondary schools, the psychological freedom of the teacher, and local geology are also included in the programme. Several well-known names are on the list of expected speakers. The Fellowship now has associated magazines in six different countries. For England, inquiries should be directed to the secretaries, New Education Fellowship, 11 Tavistock Square, London, W.C.1.

Tre Carnegie Trust for the Universities of Scotland has completed a quarter of a century's beneficent labour on behalf of Scottish education. Its activities have followed three lines : grants to the universities and extra-mural institutions, endowment of research, and assistance to students. The first are distributed in quinquennial periods, and for the years 1925-30, the twenty-fifth annual report states, there have been allocated for libraries $£ 24,225$, for buildings and permanent equipment $£ 196,500$, and for the endowment of teaching and other general purposes $\mathfrak{£} 10,500$. Research endowments for 1925-26 claimed $£ 17,130$, and 4711 students received assistance in the payment of class fees to the extent of $£ 57,212$. Apart from the encouragement given to the acquirement of knowledge in the class-room and laboratory, the Carnegie Trust most influences science through its research endowments, a very small minority of which are devoted to history and modern languages and literature. A result is apparent in the long list of more than forty contributions to scientific knowledge which have been published during the course of the year by fellows, scholars, and grantees. Three years ago a scheme of 'teaching fellowships' was instituted, which allowed the annual award of grants to university lecturers and assistants, on condition that not less than onehalf of their time was devoted to research. This scheme seems to have borne excellent results, and is to be continued in its present form for another trial year, but one of the referees directs attention to the danger that an excessive amount of teaching may be exacted from the recipient of the grant.

IN the report for 1926 of the Association of Women Science Teachers, Miss R. Stern has an interesting and valuable paper on the teaching of chemistry in the middle school. She urges that, in the practical work, each experiment should be of such a nature that it brings about a definite result, and that each group of experiments should lead to a definite conclusion. Whenever possible these experiments should be quantitative and require the setting up of apparatus which is not too difficult for the beginner, and they should be of such a nature that faulty manipulation leads to failure. At the beginning of a chemistry course an ordinary text-book is not advisable, as the results of the experiments are given, and this may prevent the class from being conscientious or even truthful. Miss Stern then describes in detail two courses which she has successfully employed. The basis of the first was the preparation of all substances used from materials met with in common life, e.g. sand, lime, slate, 'bricks, etc. Alkalis were prepared by burning beetroot and lixiviating the residue, and altogether the course appears to be a very attractive one. The second scheme is historical, and the author says that she has found this to be more satisfactory in practice than the first, since the historical development of a science is the natural development and is, above all things, a building up from the foundations. It enables the class to realise how discoveries have been made, and teaches them clearly that one wrong step may lead to hopeless confusion. Teachers will find Miss Stern's detailed syllabus of the historical course extremely suggestive and useful ; it has evidently been carefully thought out and patiently tested in the school.

No. 2999, VoL. 119] 